\title{
Effet de la fréquence de traite sur la production laitière de la vache Zébu Peulh
}

\author{
Mariétou SISSAO, Vinsoun MILLOGO* et Georges Anicet OUEDRAOGO \\ Laboratoire de Recherche en Santé et Biotechnologies Animales, Institut du Développement Rural, Université \\ Polytechnique de Bobo-Dioulasso, 01 BP 1091 Bobo-Dioulasso 01, Burkina Faso. \\ *Auteur correspondant; E-mail : paravins@yahoo.fr; Tel (+226)70 228995.
}

\section{RESUME}

L'amélioration de la productivité laitière par le métissage a eu des résultats limités dans les pays à faible rendement car les métisses résistent peu aux dures conditions climatiques. Des alternatives ont été envisagées sur la vache Zébu la mieux adaptée aux conditions du Sahel. L'objectif de cette étude était de tester l'effet de la fréquence de traite sur la productivité de la vache Zébu Peulh. Vingt-quatre (24) vaches Zébu Peulh, primipares et multipares situées entre le premier et le quatrième mois de lactation ont été réparties dans un dispositif expérimental en Carré Latin afin de tester l'effet de la fréquence de traite sur la production du lait. Les vaches étaient réparties en trois lots de huit (8) chacun correspondant à une traite $(1 \mathrm{X})$, deux traites $(2 \mathrm{X})$ et trois traites (3X) par jour. L'intervalle de traite était de 24 heures, de 11 et 13 heures et de 5, 6 et 13 heures pour respectivement une traite, deux traites et trois traites par jour. Les mesures des quantités de lait et les prélèvements d'échantillons ont été effectués une fois par semaine de juillet à décembre 2015. La spectroscopie a été utilisée pour déterminer la composition du lait (Farm Milk Analyzer, Miris AB, Suède). Les données ont été analysées au moyen du logiciel $\mathrm{R}$ et le test de Student a été utilisé pour faire les comparaisons au seuil de probabilité $\mathrm{P}<0,05$. Les résultats montrent que quand on comparait la production d'une traite à celle de deux traites, on obtenait une augmentation significative de la production de $60,6 \%$ par jour soit $+0,71$ litre. Il en était de même en comparant la production de lait d'une traite à celle de trois traites. En plus, quand on comparait la production de lait de deux traites à celle de trois traites, l'augmentation était de 84,9\% par jour soit +1,47 litres. L'augmentation de la fréquence de traite à deux et trois traites journalière améliore quantitativement la productivité de la vache sans variations notables de la composition du lait.

(C) 2016 International Formulae Group. All rights reserved.

Mots clés : Vache Zébu, production, complémentation, augmentation, lait.

\section{Effect of milking frequency on Zebu Peulh milk production}

\begin{abstract}
Milk productivity through crossbreeding gets limited results in several very low dairy production countries because hard climate conditions are touch for crossbred. Strategies have been developed to increase local Zebu cattle productivity more adapted and well spread in the Sahel Zone of Africa. The aim of the study was to test effect of milking frequency on Zebu cattle production. Twenty four (24) primiparous and multiparous Zebu cattle between first and fourth month of lactation were allocated in Latin Square experimental design in order to test effect of milking frequency on milk production. All cows were assigned in group of eight corresponding to once daily milking (1X), twice daily milking (2X) and three times daily milking (3X). Milking interval was 24 hours, 11-13 hours and 05-6-13 hours for $1 \mathrm{X}, 2 \mathrm{X}$ and $3 \mathrm{X}$ respectively. Milk volume was measured and milk samples were collected each week from july to december 2015. Milk samples were run using infra-red spectroscopy (Farm Milk Analyzer, Miris AB, Sweden). All data were
\end{abstract}


subjected to R software and the means were compared using student $\mathrm{t}$ test at the probability level of $\mathrm{P}<0.05$. The results showed milk yield increased up to $60.6 \%$ ( +0.71 litre) from once milking to twice and from once daily milking to three times a day. When milking frequency was increased from twice to three times a day, milk yield increased up to $84.9 \%$ (+1.47 litres). It was concluded that increase of milking frequency from one to two or three times a daily increase milk yield of Zebu cow without significant variation on milk composition.

(C) 2016 International Formulae Group. All rights reserved.

Keywords: Zebu cow, production, supplementation, increase, milk.

\section{INTRODUCTION}

Malgré l'important potentiel laitier du Burkina Faso, la production locale ne couvre pas ses besoins de consommation en laits et le pays est obligé d'injecter des milliards de francs CFA par an pour satisfaire les besoins de sa population (INSD, 2013). Des solutions sont toujours envisagées pour répondre aux besoins de plus en plus croissants de la population. Parmi ces solutions, il y a le croisement des vaches locales avec celles à haut rendement laitier (Sokouri et al., 2014) mais le succès de cette option s'est révélé très éphémère et seulement une infime partie des producteurs ont accès à cette technologie (Hamadou et al., 2002). En effet, l'analyse des résultats de l'insémination artificielle en Afrique subsaharienne a montré de faibles taux de réussite (Mouiche Mouliom et al., 2013). L'autre alternative est de compter sur la vache locale est qui est bien adaptée aux conditions climatiques. En effet, la baisse de la pluviométrie a permis à la race Zébu Peulh de s'étendre à tout le Burkina Faso malgré sa sensibilité à la trypanosomose et est ainsi devenu la vache locale de la majorité des producteurs traditionnels (Sawadogo, 2013). Cependant, sa capacité de production reste très faible, soit 2 à 3 litres de lait par jour en saison des pluies (Sidibé et al., 2004) mais cela peut être améliorée à 5-7 litres si le management comme la traite est bien élaborée (Millogo, 2010). En revanche, certains pensent que son potentiel génétique est tellement faible qu'on ne peut pas l'améliorer substantiellement (Belemsaga et al., 2005). Contrairement à cette opinion, on peut améliorer la productivité laitière de cette vache locale à travers la traite et ainsi contribuer substantiellement à la production annuelle nationale. La multiplication de la traite par jour fait partie des techniques d'accroissement de la productivité laitière non encore bien exploitées au Burkina Faso ou dans beaucoup de zones à faible production laitière. Cette idée est supportée par le fait que plus la fréquence de traite augmente, plus la vache produit du lait et plus elle a besoin d'aliments supplémentaires afin de combler ses besoins (Murney et al., 2015). Or, dans le cas du Zébu, les investigations sont encore très limitées dans le domaine de la traite au Burkina Faso. C'est pourquoi, pour avoir un effet immédiat sur la production de lait comme l'a conseillé McFadden et Wall (2010) et Hardin (2015), la présente étude a été envisagée pour pallier au déficit de traite des vaches Zébu Peulh. Toute chose qui limite son potentiel. L'application de cette méthode ne présentant aucune influence négative non seulement sur la santé du pis (Smith et al., 2002 ; Dahl et al., 2004 ; Wall et McFadden, 2007), la reproduction de l'animal (Patton et al., 2006) mais aussi sur la qualité du lait produit (Wall et McFadden, 2008). L'objectif de cette étude était de tester l'effet de la fréquence de traite sur la productivité de la vache Zébu Peulh à travers l'augmentation du nombre de traites journalière. La question était de savoir si la multiplication du nombre de traites par jour pouvait-elle être appliquée à la vache Zébu Peulh dans les conditions d'un élevage laitier traditionnel?

\section{MATERIEL ET METHODES \\ Le site d'étude}

L'expérimentation a été conduite pendant 06 mois de lactation de juillet à décembre 2015 dans le village de Kimidougou dans la province du Houet à $20 \mathrm{~km}$ au Nord- 
Est de la ville de Bobo-Dioulasso aux coordonnées $11^{\circ} 18^{\prime} 0^{\prime \prime}$ Latitude Nord et $4^{\circ} 13^{\prime} 60^{\prime \prime}$ Longitude Ouest. Le choix de Kimidougou a été motivé par la proximité du site expérimental et de la bonne collaboration des producteurs avec le laboratoire de recherche. En plus, ce site permettait de travailler sur le nombre de vaches Zébu Peulh exigées par l'essai en tenant compte des conditions d'élevage des fermes traditionnelles péri-urbaines du Burkina Faso.

\section{Les vaches}

L'essai a été réalisé sur 24 vaches Zébu Peulh. Une vache Zébu Peulh est caractérisée par une tête est assez longue et fine; des cornes courtes à moyenne généralement en croissant en faucille et dirigée en avant. La mamelle et les trayons sont peu développés. Les vaches étaient en début de lactation jusqu'au quatrième mois de lactation et composées de primipares et de multipares. En effet, il a été très difficile de réunir des animaux de même stade de lactation à la période de l'expérimentation. Cependant, nous avons veillé à ce que tous les stades existants soient répartis de façon homogène dans les trois lots. Le troupeau laitier constitué pour le besoin de l'expérimentation était constitué d'animaux appartenant à quatre éleveurs différents avec lesquelles un contrat été signé. Afin de contrôler au mieux les paramètres de production en milieu réel, une étable ayant les mêmes caractéristiques que celles utilisées par la plupart des producteurs traditionnels de la zone a été réalisée. L'étable était un enclos constituée de branches d'arbres et d'arbustes prélevées sur le site. Afin de garantir la propreté de l'habitat, l'enclos a été régulièrement déplacé pour éviter son humification et le dépôt très important des déjections. Les animaux étaient regroupés dans le même enclos et numéroté par traitement. Ces numéros permettaient de les différencier pendant les traitements (alimentation et traite).

\section{Le dispositif expérimental Répartition des vaches en lactation}

Le dispositif expérimental était un Carré Latin (CL) dans lequel 24 vaches Zébu Peulh (300 kg de poids vif en moyenne) en lactation étaient réparties en trois lots de huit vaches chacun. Le dispositif comportait quatre traitements à savoir, une traite, deux traites, trois traites et le complément alimentaire calculé en fonction du nombre de traites. La traite manuelle a été appliquée avec des fréquences et des intervalles de traite bien définis. Un premier lot de vaches subissait la traite une seule fois par jour (abrégé 1X) à la même période de la journée (06 heures). Un deuxième lot de vaches subissait deux traites par jour (en abrégé $2 \mathrm{X}$ ) à des périodes bien précises de la journée (traite du matin à 06 heures et traite du soir à 18 heures). Un troisième lot de vache subissait trois traites par jour (en abrégé 3X) à des périodes précises de la journée (matin 06 heures, midi à 12 heures et le à soir 18 heures). L'intervalle de traite était de 24 heures pour le traitement $1 \mathrm{X}$, de deux fois douze (12) heures pour le traitement $2 \mathrm{X}$ et de douze (12), six (06) et six (06) heures pour le traitement $3 X$.

\section{Régime alimentaire des vaches en lactation}

Les vaches étaient sous le régime alimentaire composé de l'aliment brouté sur le pâturage et du complément calculé en fonction de ce qui est reçu du pâturage et du nombre de traites appliqué. Le complément alimentaire était exclusivement du concentré (du son de maïs et du tourteau de coton). En plus du nombre de traites, la quantité de concentré a été déterminée en fonction des performances attendues pour chaque lot. Le calcul de la quantité à distribuer pour chaque lot (Tableau 1) a été effectué par la méthode de Soltner (2008) adapté à l'aide d'un prologiciel de rationnement discuté à l'atelier méthodologique du projet appui à l'amélioration durable de la productivité et de la compétitivité des filières laitières bovines en Afrique de l'Ouest et du Centre à Niamey au Niger en Février 2015. Ce pro-logiciel évalue les quantités de complément à 
distribuer en quatre étapes. Une évaluation du déficit de la ration de base (ici le pâturage naturel) en tenant compte du poids vif moyen des animaux a été effectuée. En tenant compte des données de base des deux compléments à distribuer (l'unité fourragère, la matière azotée digestible et la matière sèche) des proportions du mélange des compléments ont été calculées. Enfin, la quantité du complément à distribuer en plus de l'utilisation du pâturage a été calculée avec la valeur du mélange des compléments à distribuer en unité fourragère (UF) et en matière azoté digestible (MAD).

En fonction des quantités obtenues à partir de ce pro-logiciel, nous avons calculé l'apport nutritionnel et le coût de la complémentation en fonction de la fréquence de traites (Tableau 2).

\section{Conduite de l'alimentation par lot}

Le complément composé de tourteau de coton et de son de maïs était distribué au moment de la traite. Ainsi, le complément journalier à distribuer par traitement était repartie dans la journée en fonction du nombre de traites. Les lots traits une seule fois et deux fois par jour recevaient le complément le matin (respectivement $1,25 \mathrm{~kg}$ et $2,5 \mathrm{~kg}$ ) et celui trait trois fois par jour recevait le complément le matin $(1,5 \mathrm{~kg})$ avant le départ au pâturage et à midi $(1 \mathrm{~kg})$ au retour du pâturage. Les compléments étaient servis dans des plats individuels.

En plus du complément, les animaux recevaient du sel et de l'eau à volonté. Les lots traits une fois et deux fois par jour étaient conduits au pâturage ensemble sur une période de huit (8) heures en allez et retour. Le lot trait trois fois par jour était conduit au pâturage sur une période de quatre (4) heures en allez et retour. Chaque groupe était conduit par un berger qui lui était assigné.

\section{Traitement sanitaire et temps d'adaptation}

Pour permettre aux vaches d'exprimer le maximum de leur performance laitière, elles ont été régulièrement suivies et ont bénéficié des soins suivants :

- une injection de trypanocide (Trypanidium-Samorin) au début de l'essai ;
- une injection d'antibiotique (Oxytéracycline 20\%) au début de l'essai ;

- un déparasitage interne au Bolumisole M3 deux jours après le début de l'essai ;

- un déparasitage externe au Topline (Pour-on) ou au Vectocid une fois par mois pendant la durée de l'essai ;

- une injection d'anti-stress (Vitajec) au regroupement des animaux au cours de l'essai.

Lorsque les vaches ont été réparties de manière aléatoire et homogène entre les lots et identifiées par lot. Cette répartition se faisait en fonction de la parité et du stade de lactation. En effet, chaque lot se composait du même nombre de multipare et de primipare et de tous les stades de lactations existants. La conduite et les compléments alimentaires ont été appliqués pour une période d'adaptation de 15 jours. Tous les veaux étaient conduits ensemble par un berger et avaient accès aux compléments dans leur propre enclos pour ceux qui pouvaient en manger. Les traitements ci-dessus énumérés étaient appliqués aux vaches en lactation. C'est après cette période d'adaptation de 15 jours que la phase des mesures de lait et des prélèvements d'échantillons de lait a été introduite.

\section{Conduite de la traite du lait}

La traite des vaches était manuelle. Un trayeur était assigné à chaque lot par période de traite. En effet, les trayeurs se relayaient par période de traite dans les différents lots. Les trois trayeurs procédaient au nettoyage des mains avant chaque traite. Chaque vache était attachée à une branche et les veaux ont été utilisés pour stimuler l'éjection du lait pour toutes les vaches. Après la stimulation de l'éjection du lait, chaque veau était attaché au pied avant droit de sa mère la tête tournée vers la mamelle. Le lait de chaque vache était recueilli dans un seau individuel et la traite de chaque vache durait en moyenne (07) sept minutes.

\section{Mesures de la production et prélèvements des échantillons de lait}

Toutes les opérations de mesure du lait et des prélèvements d'échantillon de lait se faisaient une fois par semaine. La mesure de 
la quantité de lait et des prélèvements d'échantillons de lait cru a été effectuée après chaque traite de la journée. Après chaque traite, la quantité de lait par vache a été mesurée à l'aide d'un bêcher gradué. Cette estimation n'a pas tenu compte du lait consommé par le veau. La quantité journalière de chaque traitement était composée de la somme des laits produits au cours de chaque traite par jour. Ainsi, la quantité journalière du lot $1 \mathrm{X}$ n'était composé que de la quantité de lait trait le matin, celle du lot $2 \mathrm{X}$ était composé de la quantité de lait trait le matin et le soir et celle du lot $3 \mathrm{X}$ était composé de la quantité de lait trait le matin, à midi et le soir. Un échantillon de lait était prélevé après chaque traite par vache dans un flacon en plastique de 30 centilitres étiqueté et conservé dans une glacière contenant des glaçons et maintenus autour de $10{ }^{\circ} \mathrm{C}$ pour le transport au laboratoire. Compte tenu de la proximité de la ferme avec le laboratoire, la durée de transport des échantillons était de 30 minutes maximum après le prélèvement.

\section{Analyse physico-chimique du lait}

Les analyses physico-chimiques des laits prélevés ont été effectuées au Laboratoire de Recherche et d'Enseignement en Santé et Biotechnologie Animales (LA.R.E.S.B.A) à l'Université Polytechnique de Bobo-Dioulasso (UPB). Les taux de matières grasses (MG), de matières protéiques (MP), de lactose $(\mathrm{L})$, de matières sèches (MS) et de matières minérales (MM) des échantillons de laits crus ont été déterminés à l'aide de la méthode infra-rouge spectroscopie (Farm Milk Analyzer, Miris $\mathrm{AB}$, Suède). Tous les échantillons était analysés immédiatement une heure au plus après les prélèvements du jour soit une fois par semaine.

\section{Analyse statistique}

Les analyses statistiques ont été faites au moyen du logiciel $R$. Les tests de significativité ont été effectués selon le test de Student et les différences étaient considérées comme significatives au seuil de probabilité $\mathrm{P}$ $<0,05$. Les analyses univariée et bivariée ont également été effectuées. Les illustrations graphiques ont été faites à l'aide du logiciel Microsoft Office Excel 2007.

\section{RESULTATS}

\section{Effet du nombre de traites sur la quantité de lait}

Les résultats indiquent que plus le nombre de traites augmentait, plus la quantité de lait produite est importante (Tableau 3). Ainsi, la quantité de lait produite par chaque vache du lot $2 \mathrm{X}$ et $\mathrm{du}$ lot $3 \mathrm{X}$ était significativement plus élevée que celle des vaches du lot $1 \mathrm{X}(\mathrm{P}<0,05)$. En effet, lorsqu'on considère l'ensemble des vaches par lot, on s'aperçoit que les tendances sont maintenues en fonction du nombre de traites. Par exemple, le pic de production du lot $1 \mathrm{X}$ a atteint 1,2 litres, celui du lot 2X, 2,86 litres et celui du lot 3X, 3,75 litres. En plus, quand on compare la moyenne de la production par lot, les résultats montrent toujours que la production des vaches du lot $3 \mathrm{X}$ est resté toujours plus élevé pendant toute l'expérimentation par rapport respectivement au lot $2 \mathrm{X}$ et au lot $1 \mathrm{X}$.

\section{Effet de la période de traite au cours de la journée sur la quantité de lait}

Par ailleurs, le Tableau 3 indique que quand on compare la production d'une traite (1X) à celle de deux traites, on obtenait une augmentation significative de la production de $60,6 \%$ par jour soit $+0,71$ litre. En comparant la production de lait d'une traite $(1 \mathrm{X})$ à celle trois traites $(3 \mathrm{X})$ nous observons également une augmentation significative de la production. En effet, l'augmentation de la production était plus significative et la production était doublé par jour de plus de $100 \%$ soit $+2,18$ litres. Aussi, lorsqu'on compare la production de lait de deux traites $(2 \mathrm{X})$ à celle de trois traites (3X), l'augmentation suivait la même logique que dans le cas de $1 \mathrm{X}$ à $3 \mathrm{X}$ et était de $84,9 \%$ par jour soit $+1,47$ litres.

Quand on compare les quantités de lait obtenues entre les périodes de traite (Tableau 3 ), les résultats indiquent que les vaches du lot $3 \mathrm{X}$ présentaient des quantités de lait plus élevés pendant la traite du matin par rapport 
aux vaches des lots $2 \mathrm{X}$ et $1 \mathrm{X}(\mathrm{P}<0,05)$. Par contre, les vaches des lots $2 \mathrm{X}$ et $1 \mathrm{X}$ ont donné des quantités de lait similaires pendant la traite du matin $(\mathrm{P}>0,05)$. Les mêmes tendances ont été observées pour la traite du soir où les vaches des laits $3 \mathrm{X}$ ont encore des quantités plus élevées par rapport à celles des laits $2 X(P<0,05)$.

L'effet de la période de la traite au cours de la journée a été analysé en utilisant la corrélation de Pearson. Ainsi, l'analyse bivariée entre les quantités prélevées pendant la traite du matin et celle de midi présentait un coefficient de corrélation de Pearson de 0,62 $(>0,6)$. Ce qui indique une relation linéaire positive entre les périodes de traite et permet de dire que lorsque la quantité traite le matin était importante, cela présage une augmentation de la quantité qui sera traite à midi. En revanche, lorsque la quantité traite le matin était faible, celle de midi le sera également. Aussi, l'analyse bi-variée a été appliquée sur la quantité traite à midi et le soir. Dans ce cas, la corrélation de Pearson était de $0,72(>0,6)$ et permet de dire qu'il existe aussi une relation linéaire positive entre les quantités de lait obtenues à midi et le soir. Enfin, l'analyse bi-variée sur les quantités prélevées le matin et le soir a permis d'établir une corrélation de Pearson linéaire et positive de $0,77(>0,6)$. Il existe une relation linéaire entre les périodes de traite et les quantités obtenues au cours d'une journée.

Par ailleurs, lorsqu'on compare les vaches par lot, les résultats montrent que la quantité prélevée au cours de la traite du matin était toujours plus importante que celle des autres traites (midi et soir) tout lot confondu. Et, la différence était plus marquée pour le lot $3 \mathrm{X}$. Comme les vaches étaient indépendantes au sein d'un même lot et les lots aussi entre eux, le test de Khi-deux a permis de savoir que la quantité variait d'une vache à autre dans le même lot et d'un lot à autre avec une valeur de $1,329.10^{-11}(\mathrm{P}<$ $0,05)$.

\section{Effets du nombre de traites sur les paramètres chimiques du lait}

La composition du lait a présenté des valeurs similaires pour tous les paramètres analysés dans cette expérimentation (Tableau 4). Ainsi, le taux de matières grasses (MG), de matières protéiques (MP), de Lactose, de matière sèche (MS) et de matières minérales (MM) n'a pas significativement varié entre les différentes traites appliquées $(1 \mathrm{X}, 2 \mathrm{X}, 3 \mathrm{X})$. Il a été observé une augmentation non significative des taux de MG et MS et une diminution non significative du taux de MP. En plus, on note également une faible variation pour un paramètre pris individuellement excepté pour le pourcentage de MS où la variation standard a atteint plus ou moins deux.

Les effets notables $(\mathrm{P}<0,05)$ de la période de traite de la journée sur la composition chimique du lait s'observent uniquement au niveau des matières sèches et grasses pour le lot $2 \mathrm{X}$ et $3 \mathrm{X}$ (Tableau 5). Pour le lot $3 \mathrm{X}$, les taux observés à midi sont plus élevés que ceux du soir, qui à leur tour étaient plus élevées que celles du matin $(\mathrm{P}<0,05)$ au niveau des matières sèches et grasses (Tableau 5). Pour le lot $2 X$, les taux observés le soir était plus élevés que ceux du matin $(\mathrm{P}<0,05)$ au niveau des matières sèches et grasses (Tableau 5). Les taux de ces deux composants chimiques évoluent inversement proportionnels à la quantité de lait produite.

Également, nous avons observé une différence au niveau des matières protéiques, grasses et minérales du matin. En effet, à ce niveau, les taux observés le matin au niveau $\mathrm{du}$ lot $1 \mathrm{X}$ et du lot $3 \mathrm{X}$ étaient différents de ceux observés au niveau du lot $2 X$ (inférieur pour les MG et supérieur pour les MP et MM) (Tableau 5). Nous avons aussi observé une différence au niveau des matières protéiques et minérales du soir. En effet, à ce niveau, les taux observés le soir au niveau du lot $3 \mathrm{X}$ étaient inférieurs à ceux observés au niveau du lot 2X (Tableau 5).

\section{Rentabilité économique en fonction du nombre de traites et de la complémentation}

$\mathrm{Au}$ terme de l'expérimentation, un calcul de rentabilité a été fait afin de s'assurer de la cohérence économique de la méthode. Le Tableau 6 récapitule cette analyse des dépenses et des gains par type de traitement. Ainsi, nous constatons qu'en fonction de la 
production journalière moyenne du lait par vache et par traitement, nous obtenons 355 FCFA/vache pour le lot $1 \mathrm{X}, 555 \mathrm{FCFA} /$ vache pour le lot 2X et 1290/vache FCFA pour le lot $3 \mathrm{X}$. Ces sommes obtenus par vache sont exemptées du prix de la complémentation. Ce calcul de bénéfice n'a pas tenu compte du travail fournit pour la production du lait dans l'exploitation. La fréquence de deux traites rapportait 1,56 fois qu'une traite journalière ; celle de trois traites rapportait 3,63 fois plus qu'une traite journalière; et celle de trois traites rapportait 2,32 fois que deux traites journalières. La complémentation représentait entre 20 et $36 \%$.

Tableau 1 : Quantité du complément par nombre de traites en fonction du coût des ingrédients.

\begin{tabular}{lccc}
\hline Nombre de traites/J & $\mathbf{1 X}$ & $\mathbf{2 X}$ & $\mathbf{3 X}$ \\
\hline Coût (FCFA) & 155 & 310 & 310 \\
Tourteau de coton $(\mathrm{kg})$ & 0,75 & 1,5 & 1,5 \\
Son de maïs $(\mathrm{kg})$ & 0,5 & 1 & 1 \\
\cline { 2 - 3 } 1X & une traite par jour ; $2 \mathrm{X}$ : deux traites par jour et 3X : trois traites par jour.
\end{tabular}

Tableau 2 : Apport nutritionnel du complément par nombre de traites.

\begin{tabular}{lccc}
\hline & \multicolumn{3}{c}{ Nombre de traites par jour } \\
\cline { 2 - 4 } Nutriments (g) & $\mathbf{1 X}$ & $\mathbf{2 X}$ & $\mathbf{3 X}$ \\
\hline MS & 1161 & 2323 & 2323 \\
MM & 174 & 348 & 348 \\
MO & 1076 & 2151 & 2151 \\
N & 64 & 127 & 127 \\
MAT & 397 & 794 & 794 \\
NDF & 393 & 786 & 786 \\
ADF & 104 & 207 & 207 \\
\hline
\end{tabular}

MS : Matière sèche ; MM : Matière Minérale ; MO : Matière Organique ; $:$ Azote ; MAT : Matière Azotée Totale ; ADF : Acid Detergent Fiber ; NDF $\quad$ : Neutral Detergent Fiber ; g : Gramme ; $1 \mathrm{X}$ : une traite par jour; $2 \mathrm{X}$ : deux traites par jour et $3 \mathrm{X}$ : trois traites par jour. Source : (Ira, 2015).

Tableau 3 : Quantité moyenne de lait produite en $\mathrm{L} / \mathrm{j}$ et par vache et du pic de la lactation en fonction du nombre de traite par jour.

\begin{tabular}{lccc}
\hline & $\mathbf{1 X}$ & $\mathbf{2 X}$ & $\mathbf{3 X}$ \\
\cline { 2 - 4 } & \multicolumn{3}{c}{} \\
Production moyenne & $1,02 \pm 0,35^{\mathrm{a}}$ & $1,73 \pm 0,47^{\mathrm{b}}$ & $3,2 \pm 0,45^{\mathrm{c}}$ \\
Pic de lactation & $1,20^{\mathrm{a}}$ & $2,86^{\mathrm{b}}$ & $3,79 \mathrm{c}$ \\
Production de lait du matin & $1,02 \pm 0,35^{\mathrm{a}}$ & $1,07 \pm 0,54^{\mathrm{a}^{*}}$ & $1,52 \pm 0,62^{\mathrm{b}^{*}}$ \\
Production de lait de midi & - & - & $0,82 \pm 0,31^{* *}$ \\
Production du lait du soir & - & $0,68 \pm 0,40^{\mathrm{a}^{* *}}$ & $0,91 \pm 0,43^{\mathrm{b}^{* *}}$ \\
\hline 1X : une traite $; 2 \mathrm{X}:$ deux traites et $3 \mathrm{X}:$ trois traites $;$ L $/ \mathrm{j}:$ Litre par jour. \\
a, b et $\mathrm{c}:$ désigne la différence $(\mathrm{p}<0,05)$ entre les éléments d'une même ligne. \\
* et ** : désigne la différence $(\mathrm{p}<0,05)$ entre les éléments d'une même colonne.
\end{tabular}


Tableau 4 : Composition chimique moyenne du lait par nombre de traites.

\begin{tabular}{cccc}
\hline & $\mathbf{1 X}$ & $\mathbf{2 X}$ & $\mathbf{3 X}$ \\
\cline { 2 - 4 } MG (\%) & $3,73 \pm 1,38^{\mathrm{a}}$ & $3,70 \pm 1,24^{\mathrm{a}}$ & $4,15 \pm 1,02^{\mathrm{a}}$ \\
MP (\%) & $3,74 \pm 0,79^{\mathrm{a}}$ & $3,98 \pm 0,64^{\mathrm{a}}$ & $3,67 \pm 0,41^{\mathrm{a}}$ \\
L (\%) & $4,36 \pm 0,4^{\mathrm{a}}$ & $4,35 \pm 0,42^{\mathrm{a}}$ & $4,38 \pm 0,4^{\mathrm{a}}$ \\
MS (\%) & $12,98 \pm 2,2^{\mathrm{a}}$ & $13,28 \pm 2,38^{\mathrm{a}}$ & $13,58 \pm 1,73^{\mathrm{a}}$ \\
MM (\%) & $9,36 \pm 1,34^{\mathrm{a}}$ & $9,74 \pm 1,23^{\mathrm{a}}$ & $9,46 \pm 0,78^{\mathrm{a}}$ \\
\hline
\end{tabular}

Pourcentage de Matières grasses (MG); de Matières protéiques (MP); de Lactose (L); de Matières sèches (MS) ; de Matières minérales $(\mathrm{MM}) ; 1 \mathrm{X}$ : une traite ; $2 \mathrm{X}$ : deux traites et $3 \mathrm{X}$ : trois traites. $a, b$ et $\mathrm{c}$ : désigne la différence $(\mathrm{p}<0,05)$ entre les éléments d'une même ligne.

Tableau 5 : Effet de la période de traite sur la composition chimique moyenne du lait.

\begin{tabular}{|c|c|c|c|c|}
\hline $\begin{array}{l}\text { Nombre de } \\
\text { traites/Jour }\end{array}$ & & $\mathbf{1 X}$ & $2 X$ & $3 X$ \\
\hline \multirow{3}{*}{ MG (\%) } & $\mathrm{Ma}$ & $3,73 \pm 1,38^{\mathrm{a}}$ & $3,5 \pm 1,25^{\mathrm{b}^{*}}$ & $3,78 \pm 0,88^{a^{*}}$ \\
\hline & Mi & & & $4,57 \pm 1,3^{* *}$ \\
\hline & So & & $3,9 \pm 1,25^{\mathrm{a}^{* *}}$ & $4,08 \pm 1,05^{\mathrm{a}^{* * *}}$ \\
\hline \multirow{3}{*}{ MP (\%) } & $\mathrm{Ma}$ & $3,74 \pm 0,79^{\mathrm{a}}$ & $3,96 \pm 0,65^{\mathrm{b}^{*}}$ & $3,74 \pm 0,4^{\mathrm{a}^{*}}$ \\
\hline & Mi & & & $3,62 \pm 0,39^{*}$ \\
\hline & So & & $3,99 \pm 0,65^{\mathrm{a}^{*}}$ & $3,66 \pm 0,45^{\mathrm{b}^{*}}$ \\
\hline \multirow{3}{*}{$\mathrm{L}(\%)$} & $\mathrm{Ma}$ & $4,36 \pm 0,4^{\mathrm{a}}$ & $4,34 \pm 0,53^{\mathrm{a}^{*}}$ & $4,43 \pm 0,44^{\mathrm{a}^{*}}$ \\
\hline & $\mathrm{Mi}$ & & & $4,36 \pm 0,32^{*}$ \\
\hline & So & & $4,36 \pm 0,31^{\mathrm{a}^{*}}$ & $4,37 \pm 0,46^{\mathrm{a}^{*}}$ \\
\hline \multirow{3}{*}{ MS (\%) } & $\mathrm{Ma}$ & $12,98 \pm 2,21^{\mathrm{a}}$ & $12,9 \pm 2,37^{\mathrm{a}^{*}}$ & $13,11 \pm 1,41^{\mathrm{a}^{*}}$ \\
\hline & Mi & & & $14,08 \pm 2 * *$ \\
\hline & So & & $13,6 \pm 2,40^{\mathrm{a}^{* *}}$ & $13,55 \pm 1,78^{\mathrm{a}^{* * *}}$ \\
\hline \multirow{3}{*}{ MM (\%) } & $\mathrm{Ma}$ & $9,36 \pm 1,34^{\mathrm{a}}$ & $9,67 \pm 1,13^{\mathrm{b}^{*}}$ & $9,43 \pm 0,7^{\mathrm{a}^{*}}$ \\
\hline & $\mathrm{Mi}$ & & & $9,52 \pm 0,8^{*}$ \\
\hline & So & & $9,78 \pm 1,34^{\mathrm{a}^{*}}$ & $9,45 \pm 0,86^{b^{*}}$ \\
\hline
\end{tabular}

Tableau 6 : Marge bénéficiaire du lait en fonction du nombre de traite et du complément alimentaire.

\begin{tabular}{lccc}
\hline Nombre de traites/Jour & $\mathbf{1 X}$ & $\mathbf{2 X}$ & $\mathbf{3 X}$ \\
\cline { 2 - 4 } Quantité moyenne de lait (L/j) & 1,02 & 1,73 & 3,2 \\
Valeur du lait (FCFA) & 510 & 865 & 1600 \\
Valeur additionnelle de la complémentation (FCFA) & 155 & 310 & 310 \\
Valeur de la marge bénéficiaire (FCFA) & 355 & 555 & 1290 \\
Part de la complémentation sur la valeur du lait (\%) & 30,39 & 35,84 & 19,4 \\
\hline
\end{tabular}

$1 \mathrm{X}$ : une traite $; 2 \mathrm{X}:$ deux traites et $3 \mathrm{X}:$ trois traites $; \mathrm{L} / \mathrm{j}:$ Litre par jour. 


\section{DISCUSSION \\ Effet du nombre de traites sur la quantité de lait}

Les résultats de la présente étude ont permis de savoir que même dans le cas d'une vache locale et dans les conditions d'élevage traditionnel, quand on augmente le nombre de traites par jour, l'augmentation de la quantité de lait produite par vache par jour était immédiate. Cette situation est très connue et peut s'appliquer à tous les stades de lactation (Smith et al., 2002 ; Norgaard et al., 2005 ; McFadden et Wall, 2010). Dans le cas présent, les vaches étaient à des stades de lactation différents mais l'effet a été le même. Ainsi, il est possible d'améliorer la quantité de lait produite par jour à travers la traite. Ce qui est très important pour les zones à rendement laitier toujours très faible dont l'objectif central est l'augmentation de la productivité des vaches locales plus adaptées aux conditions d'élevages et au climat. Cependant, l'ampleur de la réponse diminue quand le stade de lactation augmente (Hart et al., 2013). Cependant, il faudra aussi retenir que nonobstant le stade de lactation et la race de la vache, une bonne alimentation des animaux est une condition préalable à l'accroissement de la quantité journalière de lait produite en cas de multiplication de la fréquence de traite (Murney et al., 2015). Par ailleurs, des études effectuées sur des races à fort rendement laitier ont également observé une production plus élevée dû à l'augmentation de la fréquence de traite (Smith et al., 2002; Hart et al., 2013 ; Atashi, 2015). Les présents résultats sont applicables aux fermes de production traditionnelle car la multiplication de la traite est un moyen d'augmenter instantanément la production journalière ou même saisonnière sans investissements supplémentaires au niveau de la taille du troupeau sauf sur les trayeurs dont la rémunération sera couverte par la vente du lait obtenu.

L'augmentation de la quantité de lait par jour par l'accroissement du nombre de traite est due à plusieurs facteurs. Parmi les facteurs ayant entrainés l'accroissement de la production du lait au cours la présente expérimentation, on peut citer la pression mammaire, la prolifération des cellules de sécrétion et la production des hormones (Stelwagen, 2001 ; Wall et McFadden, 2007 ; Hardin, 2015). En effet, lorsque la synthèse du lait s'accroit, la pression mammaire augmente par le remplissage de la glande et lorsque la vache n'est pas traite, cela a une conséquence sur la prochaine synthèse d'où la diminution de la quantité produite. Cette situation est récurrente dans les élevages laitiers traditionnels où ils ne font qu'une seule traite par jour. Dans ce cas d'espèce, nous avons l'effet du « feedback inhibitor » (Delamaire et Guinard-Flament, 2006) qui s'explique par l'action d'une glycoprotéine du lait ayant un rôle d'inhibiteur de la synthèse de la caséine et du lactose. C'est à travers ce phénomène que la synthèse du lait est autorégulée mais il est souvent réversible quand il s'agit d'une seule traite qui s'explique par un traitement sanitaire ou une gestation.

L'intervalle de traite joue également un rôle sur la production journalière. Différentes sources indiquent que la production de lait par vache ne peut présenter une diminution que si la limite physiologique d'un intervalle acceptable de 16 à 18 heures est atteinte (De Bie et al., 2000 ; Stelwagen et al., 2008). Dans le cas d'une traite journalière, la diminution de la production est due à l'augmentation de la pression mammaire en raison des intervalles de traites prolongées entrainant la perte de la fonction de sécrétion des cellules épithéliales mammaires (Delamaire et Guinard-Flament, 2006).

Les différences statistiques observées entre les quantités moyennes de lait prélevées dans un même lot selon la période de traite de la journée sont dues aux différents intervalles de traite observés durant l'expérimentation. Les quantités moyennes de lait produites le matin étaient supérieures à celles du midi et du soir. En effet, il existe de fortes fluctuations de la production selon que l'intervalle de traite soit court ou long (Brocard, 2007). L'intervalle de traite étant plus long (13 heures) entre la traite du soir et celle du matin fait que la quantité de lait produit le matin est supérieure à celle du midi 
et du soir. Selon les analyses statistiques, les quantités moyennes de lait prélevées le matin dans tous les lots sont dues aux traitements subis. Ainsi, les différents traitements (fréquence de traite) influencent la production de lait du matin dans chaque lot.

\section{Effet de la fréquence de traite sur la composition chimique du lait}

En générale et sur la base des moyennes obtenues, aucune différence significative n'a été observée au niveau de la composition chimique entre les différents lots. En effet, selon certains auteurs, la multiplication de la fréquence de traite ne présente aucune incidence significative sur la qualité du lait produit (Wall et McFadden, 2008; Atashi et al., 2015). Cependant, le rendement matières grasses du lait et en matières protéiques du lait augmente en raison de l'augmentation de la production de lait. Les mêmes résultats ont été observés par Soberon et al. (2011), Wright et al. (2013) et Hardin (2015) qui ont trouvé une augmentation significative du rendement en matières grasses du lait et en matières protéiques du lait.

Par ailleurs, les résultats obtenus par Millogo (2010) indiquent des valeurs supérieures à celles de la présente étude sur le pourcentage de matières grasses, de matières protéiques et de lactose aussi bien pour les laits des matins et des soirs pris séparément. Des résultats obtenus au Sénégal sur des Zébu Peulh Sénégalais (Gobra) ont également présentés des valeurs supérieurs par rapport à la présente étude aussi bien en saison froide qu'en saison chaude (Kalandi et al., 2015). Elle est d'autant plus marquée pour les laits des vaches des lots $3 \mathrm{X}$ par rapport aux lots $1 \mathrm{X}$ et 2X. Cette situation s'explique par l'augmentation de la quantité de lait qui est inversement proportionnelle aux pourcentages de matières grasses et protéiques. En d'autres termes, plus la traite se fait à des intervalles très rapprochés plus le taux des constituants est faible par unité de volume obtenu. Cependant, cette différence n'est pas aussi marquée pour tous les lots car les travaux de Millogo (2010) avaient uniquement utilisé deux traites par jour. Le taux de matières grasses (MG) et de la matière sèche (MS) des lots $2 \mathrm{X}$ et $3 \mathrm{X}$ a présenté une variation en fonction de la période de traite. En effet, les taux du midi étaient plus élevés que ceux du soir qui, à leur tour étaient plus élevés que ceux du matin. Ce qui voudrait dire que plus la quantité produite dans la journée est faible, plus le taux de MG et de MS augmente et inversement. L'intervalle de traite étant plus court entre la traite du matin et celle du midi et du soir fait que les quantités produites le midi et le soir sont inférieures à celle du matin. Il s'applique donc le principe du rapport inversement proportionnelle entre le volume du lait et les constituants du lait (Ayadi et al., 2003 ; Auldist et al., 2007). Les vaches Zébu Peulh peuvent être traites à intervalles rapprochés sans conséquences notables sur la composition du lait.

\section{Rentabilité économique en fonction du nombre de traite et du complément alimentaire}

L'augmentation de la fréquence de traite à deux et trois traites journalière est rentable en tenant compte seulement du coût additionnel de la complémentation (Tableau 6). En effet, le travail a été effectué en milieu réel ou la prise en compte du travail liée à la production est inexistante. Selon Varner et al. (2002) l'adoption de la multiplicité de la fréquence de traite journalière doit passer par une évaluation de la rentabilité. En effet, la traite plus fréquente n'est rentable que lorsque la valeur du lait supplémentaire produit dépasse les coûts associés aux traites supplémentaires (McFadden et Wall, 2010 ; Hardin, 2015). Selon nos résultats, la valeur $\mathrm{du}$ lait obtenu à deux et trois traites journalières dépasse les coûts additionnels dus aux traites supplémentaires. La complémentation journalière dans notre étude en plus du pâturage a représenté une valeur de 20 à $36 \%$ de la valeur journalière du lait produit par vache. Les animaux du lot trait trois fois par jour ont obtenu le meilleur pourcentage de $19,4 \%$. Ce qui nous permet de dire que la fréquence de trois traites journalière a été plus efficace que les autres fréquences. Ainsi, plus on augmente la 
fréquence de traite, plus le rapport entre l'alimentation et la production est meilleure. Au-delà du bénéfice financier engendré, il convient toutefois de tenir compte du travail supplémentaire engendré pour une décision plus profitable car selon Davis et al. (2008), la traite représente environ $50 \%$ des activités d'une ferme laitière, ce qui n'est pas négligeable comme charges.

\section{Conclusion}

Cette expérimentation a permis de démontrer que la multiplication de la traite journalière peut être applicable à la vache Zébu Peulh dans les conditions des fermes de production traditionnelle. Cependant, une meilleure adaptation de la vache est toujours nécessaire dans les conditions de stabulation plus contrôlées. L'application de deux et trois traites journalière a permis d'augmenter significativement la production laitière journalière ou même saisonnière des vaches Zébu Peulh sans investissements supplémentaires au niveau de la taille du troupeau. Néanmoins, ceci a occasionné un travail supplémentaire pour les trayeurs dont la rémunération devra être couverte par la vente du lait obtenu. Les vaches Zébu Peulh peuvent être traite à intervalles rapprochés sans conséquences notables sur la composition du lait. L'augmentation de la fréquence de traite à deux et trois traites journalière est rentable en tenant compte seulement du coût additionnel de la complémentation. Au-delà $\mathrm{du}$ bénéfice financier engendré, il convient toutefois de tenir compte du travail supplémentaire engendré pour une décision plus profitable.

\section{REMERCIEMENTS}

Nos remerciements s'adressent au Laboratoire de Recherche en Santé et Biotechnologies Animales (LA.RE.S.B.A) qui a abrité toutes les analyses et ayant supervisé les travaux de terrain. Notre reconnaissance va également aux éleveurs du village de Kimidougou (Bobo-Dioulasso, Burkina Faso) qui nous ont permis de travailler sur leurs animaux ainsi qu'à Monsieur Abel
DEMBELE pour son aide au cours de l'expérimentation.

\section{CONFLIT D'INTERETS}

Les auteurs déclarent ne pas avoir de conflits d'intérêts en relation avec cet article.

\section{CONTRIBUTIONS DES AUTEURS}

MS a été responsable de l'expérimentation, de l'exploitation des résultats et de la rédaction du manuscrit. VM a validé le protocole expérimental, supervisé les travaux et a participé à la rédaction du manuscrit. Il a été le responsable scientifique de cette étude. GAO a contribué à l'amélioration du manuscrit.

\section{REFERENCES}

Atashi H. 2015. Effects of milking frequency on the lactation performance an lactation curve of Hostein dairy cows in Iran. Iranian Journal of Applied Animal Science, 5(2): 273-278.

Auldist MJ, O'Brien G, Cole D, Macmillian KL, Grainger C. 2007. Effect of varying lactation length on milk production capacity of cows in pasture-based dairying systems. Journal of Dairy Science, 90(7): 3234-3241.

Ayadi M, Caja G, Such X, Knight CH. 2003. Use of ultrasonography to estimate cistern size and milk storage at different milking intervals in the udder of dairy cows. J. Dairy Res., 70:1-7.

Belemsaga DMA, Lombo Y, Thevenon Sylla S. 2005. Inventory Analysis of West African Cattle Breeds. Applications of Gene-Based Technologies for Improving Animal Production and Health in Developing Countries, 167-173.

Brocard V. 2007. Réduire l'intervalle entre deux traites. Cap Elevage, 16: 10-11.

Dahl GE, Wallace RL, Shanks RD, Lueking D. 2004. Hot topic: Effects of frequent milking in early lactation on milk yield and udder health. Journal of Dairy Science, 87(49): 882-885.

Davis KL, Fulkerson WJ, Garcia SC, Dickeson D, Barchia, I. 2008. Premilking teats preparation for Australian pasture- 
based cows milked by an automated milking system. Journal of Dairy Science, 91: 2604-2609.

De Bie L, Berger YM, Thomas DL. 2000. The effect of three times a day milking at the beginning of lactation on the milk production of East Friesian crossbred ewes In Dairy sheep symposium, p. 9-17.

Delamare E, Guinard-Flament J. 2006. Longer milking intervals alter mammary epithelial permeability and the udder's ability to extract nutrients. Journal of Dairy Science 89(6): 2007-2016.

Hamadou S, Kamuanga M, Marichatou H, Kanwé A, Sidibé A, Paré J. 2002. Diagnostics des élevages périurbains de production laitière : Typologie des élevages de la périphérie de BoboDioulasso. Programme Concerté de Recherche-Développement sur l'Elevage en Afrique de l'Ouest (PROCORDEL). Etudes socioéconomiques. Document de travail $\mathrm{N}^{\circ} 1$. pp 54.

Hardin D. 2015. Local regulation of increased milk yield due to early location increased milking frequency. Master of Science in Dairy Science, Faculty of the Virginia Polytechnic Institute and State University, 62p.

Hart KD, McBride BW, Duffield TF, DeVries TJ. 2013. Effect of milking frequency on the behavior and productivity of lactating dairy cows. J. Dairy Sci., 96 : 6973-6985.

Ira M. 2015. Optimisation de la production laitière et réduction de l'intervalle vêlagevêlage chez la vache dans la zone périurbaine de Bobo-Dioulasso. Mémoire de Master en productions et industries animales, Institut du Développement Rural/Université Polytechnique de BoboDioulasso (Burkina Faso), $100 \mathrm{p}$.

ISND. 2013. Note d'analyse des indices du commerce extérieur du Burkina Faso. Quatrième trimestre 2012. Ministère de l'Economie et des Finances du Burkina Faso. 29p.

Kalandi M, Sow A, Guigma WVH, Zabre MZ, Bathily A, Sawadogo GJ. 2015. Evaluation de la qualité nutritionnelle du lait cru dans les élevages traditionnels de
Kaolacl au Sénégal. Int. J. Biol. Chem. Sci., 9(2): 901-909.

McFadden T, Wall E. 2010. Managing Milking frequency. WCDS Advances in Dairy Technology, 22: 35-47.

Millogo V. 2010. Milk Production of HandMilked Dairy Cattle in Burkina Faso. Doctoral Thesis No. 2010: 4, ISSN 16526880 ISBN, 978-91-576-7481-4, Uppsala, Sweden.

Mouiche Mouliom MM, Sow A, Kalandi M, MPouam SE, Ouedraogo GA, Sawadogo GJ. 2013. Analyse du profil protéique chez des vache Zébu Gobra artificiellement inséminées au Sénégal. Int. J. Biol. Chem. Sci., 7(2): 780-789.

Murney R, Stelwagen K, Wheeler TT, Margerison JK, Singh K. 2015. The effects of milking frequency in early lactation on milk yield, mammary cell turnover, and secretory activity in grazing dairy cows. J. Dairy Sci. 98: 305-311. http://dx.doi.org/ 10.3168/jds.2014-8745.

Norgaard, J., Sorensen, A., Sorensen, M. T., Andersen, J. B. \& Sejrsen, K. (2005). Mammary cell turnover and enzyme activity in dairy cows: Effects of milking frequency and diet energy density. $J$. Dairy Sci., 88:975-982.

Patton J, Kenney DA, Mee JF, O'Mara FP, Wathes DC, Cook M, Murphy JJ. 2006. Effect of milking frequency and diet on milk production, energy balance, and reproduction in dairy cows. J. of Dairy Sci., 89(5): 1478-1487.

Sawadogo A. 2013. Analyse de la stratégie de diffusion de Zébu Azawak du projet BKF / 017 (Burkina Faso). Mémoire de fin d'étude, Institut du Développement Rural. Option Elevage. 57 pages.

Sidibé M, Boly H, Lakouetene T, Leroy P Bosma RH. 2004. Characteristics of periurban dairy herds of Bobo-Dioulasso, Burkina Faso. Tropical Animal Health and Production, 36: 95-100.

Smith JW, Ely LO, Graves WM, Gilson WD. 2002. Effect of Milking $3 \mathrm{X}$ on DHI Performance Parameters. The Univ. of Georgia, CAES, Dept. of Animal \& Dairy Sci., Annual Report, pp. 187-92. 
Soberon F, Ryan CM, Nydam DV, Galton DM, Overton TR. 2011. The effects of increased milking frequency during early lactation on milk yield and milk composition on commercial dairy farms. J. Dairy Sci., 94(9): 4398-4405.

Sokouri DP, Gbodjo ZL, N'Goran E, Soro B. 2014. Performance de reproduction et production laitière de croisés Montbeliarde X N'Dama du «projet laitier Sud» (Côte d'Ivoire). Int. J. Biol. Chem. Sci., 8(3): 925-936.

Soltner D. 2008. Alimentation des animaux domestiques. Les principes de l'alimentation pour toutes les espèces. Collection Sciences et Techniques Agricoles (22 $2^{\text {ème }}$ édition). Paris, 176 pages.

Stelwagen K, Farr VC, Nicholas GD, Davis SR, Prosser CG. 2008. Effect of milking interval on milk yield and quality and rate of recovery during subsequent frequent milking. Livestock. Science, 114: 176180.

Stelwagen K. 2001. Effect of milking frequency on mammary functioning and shape of the lactation curve. J. Dairy Sci., 84(ESuppl.): E204-E211.

Varner M, Hale S, Capuco T, Sanders A, Erdman R. 2002. Increasing Milking Frequency. Advances in Dairy Technology, 14: 265p.

Wall EH, McFadden TB. 2007. The milk yield response to frequent milking in early lactation of dairy cows is locally regulated. Journal of Dairy Science, 90(2):716-720.

Wall EH, McFadden TB. 2008. Use it or lose it: enhancing milk production efficiency by frequent milking of dairy cows. Journal of Animal Science, 86(13 Suppl): 27-36.

Wright JB, Wall EH, McFadden TB. 2013. Effects of increased milking frequency during early lactation on milk yield and udder health of primiparous Holstein heifers. J. Anim. Sci., 91: 195-202. 